\title{
ECONOMÍAS COLABORATIVAS: PROPUESTA DE METODOLOGÍA DE IDENTIFICACIÓN Y REGULACIÓN EN MÉXICO*
}

\author{
COLLABORATIVE ECONOMIES: PROPOSAL \\ FOR IDENTIFICATION METHODOLOGY AND REGULATION \\ IN MEXICO
}

\author{
LES ÉCONOMIES COLLABORATIVES: PROPOSITION \\ DE MÉTHODOLOGIE D'IDENTIFICATION \\ ET RÉGLEMENTATION AU MEXIQUE
}

Amada María ARLEY ORDUÑA*

\begin{abstract}
RESUMEN: El Estado de derecho mexicano requiere establecer un concepto jurídico legal que describa de forma sustancial las "economías colaborativas" con la finalidad de incentivar las verdaderas economías colaborativas basadas en el bien común, las cuales ayudan a la solución de conflictos sociales. Esto permitiría clasificar, sistematizar y regular las actividades de empresas online que no contienen elementos de la "economía colaborativa" de forma tal que respondan de sus prácticas operativas.

Para demostrar dicha hipótesis, el presente trabajo primeramente dotó un marco teórico que ayudara a identificar los elementos que constituyen una economía colaborativa de los que no; como segundo paso, optó por desarrollar una metodología que permitiera arrojar la clasificación de las plataformas a partir de la actividad que desarrollan; lo que permitió el tercer paso, elaborar una propuesta de regulación en México, y, final-
\end{abstract}

* Recibido el 15 de abril de 2019 y aceptado para su publicación el 6 de septiembre de 2019.

* Candidata a doctora en derecho por la UNAM. Fundador y ex coordinador del Centro de Negociación y Mediación de la Facultad de Derecho, UNAM. Graduado del Programa de Negocios de la Facultad de Derecho en Harvard.

Revista Latinoamericana de Derecho Social
Núm. 30, enero-junio de 2020, pp. 3-35 
mente, se justificó la importancia de dichas reformas desde la dimensión social y económica actual en el país.

Palabras clave: economía, empresas, colaborativo, regulación, social.

ABSTRACT: The Mexican Rule of Law requires establishing a legal concept that describe in a substantial way the "collaborative economies" in order to encourage true collaborative economies based on the common good, which help in the solution of social conflicts. This would allow to classify, systematize and regulate the activities of online companies that do not contain elements of the "collaborative economy" in such a way that they respond to their operational practices.

To demonstrate this hypothesis, the present paper first provided a theoretical framework to help identify the elements that constitute a collaborative economy of those who do not; as a second step, it chose to develop a methodology that allowed to show the classification of the platforms based on the developed activity; what allowed the third step, to elaborate a regulation proposal in Mexico, and finally the importance of these reforms was justified from the current social and economic dimension in the country.

Keywords: Economy, business, collaborative, regulation, social.

RÉSUMÉN: L'état de droit mexicain requiert établir un concept juridique légal qui décrit d'une manière substantiel "les économies collaboratives" dans le but de promouvoir les vrais économies collaboratives fondées sur le bien commune, lesquelles aident á la résolution de problèmes sociaux. Cela permettrait classifier, systématiser, et réglementer les activités de sociétés online qui ne contiennent pas d'éléments "économie collaborative", d'une manière qu'ils répondent de leurs pratiques collaboratives.

Pour démontrer cette hypothèse, ce travail premièrement a fourni un cadre théorique cela aidera à identifier les éléments qui constituent un économie collaborative de ceux qui ne le font pas; dans un deuxième temps, a choisi de développer une méthodologie qui a permis de donner la qualification des quais à partir de l'activité qu'ils font; ce qui a permis la troisième étape, élaborer une proposition de règlementation au Mexique, et finalement c'était justifié l'importance de ces réformes á partir de la dimension social et économique actuel dans le pays.

Mots-clés: Economie, les entreprises, collaborative, règlement, social. 
SumARIO: I. Introducción. II. Economía colaborativa. III. Método para identificar una economía colaborativa. IV. Propuesta de regulación de la economía colaborativa en México. V. Dimensión social de la economía colaborativa en México.

VI. Conclusión. VI. Bibliografía.

\section{INTRODUCCIÓN}

$\Psi$ n la actualidad, empresas como Uber y Airbnb se jactan de su capacidad para establecer esquemas de negocios a partir de la financiarización de la vida cotidiana sin necesidad de infraestructura física propia. Logrando establecer monopolios millonarios a costa de activos fijos de las personas que asumen el costo del desgaste de sus propiedades, denominándolos "socios". Estos socios o colaboradores actúan bajo fe ciega en aquellos modelos de negocios y la Internet, pero reciben ínfimas ganancias en comparación con el costo que absorben del servicio que proporcionan y la cuota que deben pagar a dichas plataformas, generándose un desbalance desproporcionado de la relación.

Dichos modelos de negocios corporativos se denominan equivocadamente "economías colaborativas" cuando realmente desarrollan actividades comerciales con fines de lucro desmedido sin contrapesos de responsabilidad social o autorregulación; aprovechándose de la ignorancia de las personas, el desarrollo tecnológico, las vaguedades legales y las fallas del Estado de derecho.

Por ello, el Estado de derecho mexicano requiere establecer un concepto jurídico legal que describa de forma sustancial las "economías colaborativas", ello con la finalidad de incentivar a las verdaderas economías colaborativas basadas en el bien común, las cuales ayudan a la solución de conflictos sociales. Además, lo anterior permitiría clasificar, sistematizar y regular las actividades de empresas online que no contienen elementos de la "economía colaborativa" de forma tal que respondan de sus prácticas operativas.

Para demostrar esta hipótesis, primero se dotó de un marco teórico que ayudara a identificar los elementos que constituyen una economía colaborativa de los que no; segundo, se desarrolló una metodología que arrojará la clasificación de las plataformas a partir de la actividad que desarrollan; tercero, se elaboró una propuesta de regulación en México, y cuarto, se justificó la importancia de ello desde la dimensión social y económica actual en el país. 


\section{ECONOMÍA COLABORATIVA}

En la academia y en la praxis existen posiciones contrapuestas con respecto al concepto "economía colaborativa". Se ha hecho un abuso indiscriminado del término para definir un sinfín de plataformas que son todo menos colaborativas.

Por lo cual, he decido dilucidar el citado concepto bajo dos enfoques, primero, en cuanto a su naturaleza tecnológica Peer to Peer (P2P), y segundo, en cuanto a la actividad y finalidad de las plataformas P2P, mismas que subdivido en categorías correspondientes a dos concepciones ideológicas: la capitalista salvaje y la orientación al bien común (sin lucro o con lucro equitativo).

\section{REDES Peer to Peer (P2P).}

Las redes Peer to Peer (P2P) ${ }^{1}$ son la base tecnológica de las economías colaborativas, ya que representan el modo de comunicación entre los usuarios. De forma tal que todos los nodos son clientes y servidores, y son funcionalmente iguales. $^{2}$

Es decir, el esquema vertical (productor-consumidor) se cambia por un esquema lateral, en donde el consumidor se vuelve productor (prosumidor) logrando comunicarse con otros de su mismo nivel. Contrario al paradigma usual en el que el cliente se comunica con el servidor o proveedor de un servicio y éste a su vez le responde.

Las redes P2P pueden clasificarse en dos tipos, ${ }^{3}$ esto es: a) puras: todos los participantes son iguales y cada persona juega el rol de cliente y servidor, ${ }^{4} \mathrm{O}$ b) híbridas: puede haber un servidor centralizado que ayuda a coordinar los intercambios entre las personas.

Sin embargo, esta estructura de comunicación tecnológica igual-a-igual, no debe confundirse por su naturaleza informática como colaborativa en térmi-

1 Oram Andy, Peer to Peer: Harnessing the Power of Disruptive Technologies, marzo de 2001, p. 8, disponible en: goo.gl/Swk.GFl.

2 Bonilla Egido, Antonio y Meler Playan, Javier, “Aplicaciones Distribuidas: P2P”, Conceptes avancats de sistemes operatius, Departament d'Arquitectura de Computadors, disponible en: goo.gl/ wolloX.

3 Peer to Peer Network, Gobierno de Hong Kong, Administración especial de la región, febrero de 2008, disponible en: goo.gl/7xZjTm.

4 Por ejemplo, Gnutella y Freenatare, se pueden consultar en: https:/ / freenetproject.org/. 
nos económicos, mucho menos jurídicos, ni sociales. Ya que la determinación de "colaborativa" la dictan en términos sustanciales (no retóricos) los valores y principios de la empresa que maneja la Red P2P y oferta el servicio o uso de bienes. En tanto que son los fines, objetivos, intereses económicos y sociales que persigue ese agente económico, los que permiten comprender la corriente ideológica elegida, y con ello determinar el contrapeso que ha de realizar el Estado de derecho social.

\section{Clasificación de las economías colaborativas de acuerdo con la finalidad de la actividad}

Para comprender la esencia de la economía colaborativa debe acudirse a las raíces lingüísticas, económicas, sociales e ideológicas de esta acepción; las cuales pareciesen hablar de lo mismo, pero en el análisis de su espíritu se encuentran divergencias significativas.

Para tal efecto es prudente comenzar por atender el concepto "economía colaborativa" desde la semántica. El concepto "economía”, del latín medieval economia, goza de varias acepciones según el Diccionario de la Real Academia, se tomará la de carácter general: "administración eficaz y razonable de los bienes." 5

El concepto "colaborar", del latín collaborāre, en su carácter general lo define como "trabajar con otra u otras personas en la realización de una obra". ${ }^{6}$ Mientras que el concepto "cooperar", del latín tardío cooperāri, es definido como "obrar juntamente con otro u otros para la consecución de un fin común".?

Ambos términos se refieren al trabajo y acción para lograr una obra o fin común; entonces, se puede decir que a grosso modo, que la economía colaborativa en términos lingüísticos se refiere a la administración eficaz y razonable de los bienes que se ponen a trabajar conjuntamente entre personas para la realización de una obra cuya consecución tiene un fin común.

Aquellos que activan esta economía deben definir los valores ideológicos que habrán de seguir para la consecución de sus actividades, por lo tanto, se encontrarán en presencia dos corrientes ideológicas a elegir:

- La concepción capitalista salvaje de lucro desmedido, cuyo objetivo radica en amasar fortunas de dinero en manos de unos a costa de otros; o

\footnotetext{
5 Diccionario de la Real Academia de la Lengua Española, disponible en: goo.gl/yWimis.

6 Ibidem, disponible en: goo.gl/knBeNr.

7 Ibidem, disponible en: goo.gl/FNIWYK.
} 
- La concepción de la colaboración orientada al bien común, que a su vez está divida entre aquellos que consideran el lucro significativo o aquellos que no persiguen fines de lucro. En ambas, el objetivo radica en colaborar para ayudar a solucionar una necesidad social o bien común y que en caso de generar ganancias se distribuyen de forma transparente, justa y equitativa entre los involucrados.

\section{A. El capitalismo salvaje y las REDES Peer to Peer (P2P): "Economias corporativas online P2P"}

La estructura de intercomunicación tecnológica P2P ha sido aprovechada por unos cuantos bajo la corriente ideológica de capitalismo salvaje para crear modelos de negocios con estructuras que lejos de democratizar la participación en los mismos, crear empleos o incentivar la economía, lucran con los activos fijos subutilizados de particulares, quienes arriesgan su capital, tiempo, habilidades y propiedades en proveer servicios de los cuales reciben un beneficio desproporcionalmente bajo al riesgo que realizan.

Tom Slee en su libro Lo que es tuyo es mío, Jhon Duda en la conferencia "Cooperativismo de plataforma" y Trebor Scholz en "Cooperativismo de plataforma", coinciden con estos puntos de vista.

Así, empresas como Uber, Airbnb y Amazon, bajo la conceptualización capitalista de colaboración se autodenominan "economías colaborativas" so pretexto de una necesidad social frente a la taza desempleo más alta en la historia de la humanidad. Este tipo de "economías colaborativas" que de colaborativas sólo usan el nombre, utilizan la figura de "socio"10 o "contratista independiente"11 para eludir la figura del "empleo" y no otorgar las prestaciones mínimas de trabajo digno estipuladas por la Organización Internacional de Trabajo (OIT).

No obstante, los promotores de estas economías colaborativas, como Hon Matthew Hancock (Ministro de Negocios de Inglaterra ${ }^{12}$ ) y Debbie Wosskow

8 Scholz, Trebor, Cooperativismo de plataforma. Desafiando la economía colaborativa corporativa, En Defensa del Software Libre, 2017, p. 11 en: https:/ / endefensadelsl.org. También se puede consultar video en Entrevista, Jhon Duda, Plataform Cooperativism, The Internet, Ownership, democracy, the new schoool, 13-14 de noviembre, disponible en: https:/ / vimeo.com/149401422.

9 Idem.

10 Uber, Contratos para socios, disponible en: goo.gl/jHV ${ }^{5} 5 \mathrm{Z}$.

11 Airbnb, Términos y condiciones legales, disponible en: goo.gl/ZAi9rg.

12 Algunas opiniones vertidas como la del primer ministro de estado de negocios, empre- 
(sector privado) ostentan argumentos de defensa basados en incentivar la economía en tiempos de desempleo, sostienen que todas las personas pueden rentar, alquilar u ofrecer servicios con sus bienes, propiedades o activos fijos y generar ganancias a partir de activos que permanecían infrautilizados. ${ }^{13}$

En esta corriente de ideas también se encuentran varios académicos. ${ }^{14}$ Lo que no dice Wosskow y muchos autores, es que mientras la empresa dueña de la plataforma P2P se enriquece a un nivel descomunal, el particular obtiene una ganancia pequeña y un alto costo de depreciación y desgaste de su propiedad.

Los defensores de estas economías, políticamente se jactan de generar empleos o modalidades flexibles de trabajo, lo cual es una clara falacia, ya que en sus contratos las cláusulas son contundentes respecto a resaltar que los firmantes no son empleados y maliciosamente los denominan contratistas independientes.

La confusión conceptual es tan grave que inclusive el Comité Económico y Social Europeo (CESE) en su dictamen elaborado en 2015 subraya que las economías colaborativas "generan ingresos más allá de las relaciones de empleo lineales tradicionales y hace posible que las personas trabajen con arreglo a modalidades flexibles". ${ }^{15}$

Es decir, lo presenta como una oportunidad positiva en el sentido que las personas deben estar agradecidas por tener un empleo flexible, o por cuenta propia. Sin embargo, desarrollar una actividad flexible ¿significa no tener derecho a prestaciones sociales?

Para desmitificar ese supuesto, la sentencia de un juez de California reconoce que Barbara Ann, chofer de Uber, era empleada y no contratista in-

sas y energía de Reino Unido, describen a las economías colaborativas como nuevas, destaca que la gente está haciendo dinero de estos nichos, trabajos o habilidades, palabras que se citan de forma textual en su idioma original. Rt Hon Matthew Hancock MP Minister of State for Business, Enterprise and Energy. Esta declaración se puede encontrar en el Preámbulo de Debbie Wosskow, Unlocking the Sharing Economy, Reporte Independiente, Department for Business, Innovation \& Skills, Reino Unido, 2014, p. 2, disponible en: goo.gl/yJmjDL.

13 Ibidem, p. 7.

14 En el libro The Sharing Economies, se puede encontrar en su contenido una asociación de estos modelos de negocio al concepto economía colaborativa; se define en general a la economía colaborativa como nueva, y exitosa. Farronato Chiara, Levin Jonathan, "The Rise Peer to Peer Bussines", The Sharing Economies, Global Investor 2.15, noviembre de 2015, pp. 8-11.

15 Comunicación de la comisión al parlamento europeo al consejo al comité económico y social europeo y al comité de las regiones, Una Agenda Europea para la economía colaborativa, Versión final, Bruselas, 2016, disponible en: goo.gl/CSF77r (fecha de consulta: 4 de mayo de 2017). 
dependiente. ${ }^{16}$ Misma que ha servido de antecedente para que choferes de Uber de California interpusieran una demanda colectiva contra la empresa. El juicio aún se desarrolla y Uber ha apelado. ${ }^{17}$

Transpolando el supuesto a la legislación laboral mexicana, debe seguirse la línea de pensamiento en la que no es necesario que se establezca un contrato de trabajo para que exista la relación laboral, basta comprobar una relación de subordinación y forma de pago para comprobar dicha relación. De forma tal que, el caso de Uber tendría la misma consecuencia bajo ese supuesto lógico jurídico.

Estas empresas en realidad son estructuras clásicas de administración piramidal y corporativa que evaden obligaciones y responsabilidades legales en diversas materias: laboral, protección al consumidor, resolución de conflictos, protección de datos personales, fiscal, etcétera, por mencionar algunas.

Scholz las califica como "economías colaborativas corporativas", 18 aunque resulta más preciso llamarlas por la sustancia de su actividad y no por la falacia con la que se designa su actividad, v.g., economías corporativas online P2P.

A consecuencia de sus actividades, estas empresas corporativas online P2P pueden llegar a cotizar en la bolsa y obtener riqueza por especulación financiera, tal como ya lo hace Amazon o Airbnb (proyección) con pleno derecho. Lo incongruente e inmoral radica en que se autocalifiquen como "economías colaborativas" y no sean socialmente responsables con quienes trabajan y ponen en riesgo sus propios recursos, para el goce y disfrute de esa riqueza.

Scholz Trebor ha realizado entrevistas en Estados Unidos a "socios" de Uber, y repetidamente reportan que no es un buen negocio, ya que el desgaste y depreciación de la propiedad es muy alto, así como el tiempo de cuidar el negocio; según su dicho, para que sea un "buen negocio" se requieren por lo menos cuatro autos. ${ }^{19}$ Dichos reportes coinciden con entrevistas aisladas que he realizado con el motivo del presente trabajo en México. Lo cual indica que el

16 Isaac, Mike y Singer, Natasha, "California Says Uber Driver Is Employee, Not a Contractor", New York Times, 17 de junio de 2015, disponible en: goo.gl/1q5rJr (fecha de consulta: 26 de enero de 2017).

17 Se puede consultar el sitio de la demanda colectiva. Disponible en http:// uberlawsuit. $\mathrm{com} /$.

18 Scholz Trebor, Cooperativismo de plataforma. Desafiando la economía colaborativa corporativa, Barcelona, Publicaciones Dimmons, Universitat Oberta de Catalunya, 2016, p. 6, disponible en: goo.gl/TN8u1S.

19 Idem. 
modelo de negocio es igual de desproporcional y salvaje independientemente de la zona geográfica en que opere.

Así, el capitalismo salvaje se ha ayudado de las tecnologías de la información para crear modelos económicos que generan nichos de mercado de contacto masivo, logrando beneficios lucrativos excesivos a costa de activos fijos de individuos que deben asumir el desgaste, es decir, un capitalismo salvaje automatizado. En suma, las empresas corporativas online P2P son en realidad modelos tradicionales de empresas, no un modelo colaborativo de ayuda mutua, y requieren comprenderse así para la regulación de los mismos con la finalidad de desarrollar una fiscalización justa y promover la responsabilidad social con sus socios particulares.

\section{B. "Economías colaborativas orientadas al bien común"}

vistas desde la economía social

En sentido estricto, la economía colaborativa no es nueva, sus antecedentes se pueden rastrear hasta el trueque. El concepto "sociedades cooperativas" del siglo XIX se puede considerar un antecedente de las economías colaborativas en la era tecnológica del siglo XXI, ya que ambas se basan en la idea de la colaboración.

La correlación y comparación entre sociedades cooperativas y economías colaborativas resulta pertinente dado que ambas se determinan por anteponer el bien común al capital, principio subyacente de la economía social.

Las cooperativas en un contexto económico se han utilizado como un recurso social en respuesta a una problemática que afecta a una comunidad específica en un sector determinado, y algunas veces como parte del Estado de derecho social.

Ejemplo de ello se denota en los registros históricos con la cooperativa de Rochdale ${ }^{20}$ En estas, los trabajadores se unen para competir en la industria bajo un esquema de administración igualitaria, justa y equitativa de repartición de utilidades y dividendos. ${ }^{21}$

20 En 1844 en Inglaterra, artesanos tejedores decidieron vender mercancías, unificar los precios y que las ganancias obtenidas se dividieran entre todos, famosamente conocida como la cooperativa fundada en Rochdale. En 1854 en Italia, varios trabajadores se unieron en la cooperativa "La Proletaria". Se puede consultar más en Mladenatz, Gromoslav, Historia de las doctrinas cooperativas, cooperativas, trad. de Carmelo Tumino, Buenos Aires, Intercoop, 1969, pp. 63-65.

21 Charterina Martinez, Alejandro, "Los valores y principios cooperativos", Dialnet, p. 4, disponible en: goo.gl/TBW qeh. 
Por su parte, las economias colaborativas orientadas al bien común surgen también a partir de una necesidad social que afecta a una comunidad, siendo los desarrolladores tecnológicos los que captan esa necesidad y le dotan impacto masivo a través de la programación de redes y la Internet (soporte tecnológico con Redes P2P). Es decir, ayudan a conectar a la comunidad para que ella misma satisfaga una necesidad determinada. De tal forma, por esta actividad el desarrollador tecnológico puede tener un pago justo, lucro justo o no tener fines de lucro. Algunos ejemplos son:

- Blablacar, empresa que actúa como intermediario tecnológico entre particulares para facilitar asientos de transporte en viajes cortos o largos, se promueve un pago justo y equitativo sin fines de lucro y sólo para compartir gastos del viaje entre los particulares. La plataforma cobra gastos de gestión. $^{22}$

- Tutanda, plataforma mexicana que dota de un sistema electrónico para hacer y administrar tandas gratuitamente, sin tener contacto con el dinero, establece dos formas de realizar las contribuciones: una fuera de línea y la otra con datos bancarios. ${ }^{23} \mathrm{Y}$ no cobra gastos de administración.

- Mytwinplace, plataforma española que pone en contacto a viajeros para intercambiar habitaciones, para ello crea un sistema de puntos a fin de gestionar el intercambio, éste sistema no tiene un valor económico ni se traduce en valor monetario. ${ }^{24}$ Además, no establece cobro de gestión.

- La economía colaborativa de forma pura se encuentra en la práctica del Blockchain ${ }^{25}$ y del Código abierto ${ }^{26}$ o Software libre. Éstas permiten el desarrollo de cooperación a un "Costo Marginal Cero", es decir, que

22 Blablacar, Términos y condiciones, disponible en: goo.gl/ZOkhbN.

23 Tutanda.com, Términos y condiciones, disponible en: goo.gl/FSL ₹ $9 j$.

24 Mytwinplace.com, Términos y condiciones, disponible en: goo.gl/KnFGxx.

25 Satoshi Nakamoto, Bitcoin: A Peer-to-Peer Electronic Cash System, disponible en: https:// bitcoin.org/bitcoin.pdf. También se puede consultar: wmw.bitcoin.org, Fairfield Joshua, A. T., "Smart Contracts, Bitcoin Bots, and Consumer Protection”, 71 Wash. \& Lee L. Rev. Online 36 (2014), disponible en: goo.gl/GfEpGY.

26 Código abierto, significa tener la libertad de modificar el programa para satisfacer sus necesidades, con un código de acceso al código fuente, para realizar cambios; libertad de redistribuir copias gratis o por una tarifa, distribuir versiones modificadas del programa, ello para que la comunidad pueda beneficiarse de sus mejoras. Para más información, consultar: Stallman Richard, "The GNU Operating System and the Free Software Movement", Open Sources: Voices from the Open Source Revolution, enero de 1999, disponible en: goo.gl/6uA2XG. 
las personas obtienen beneficios a un costo nulo. ${ }^{27}$ Lo cual rompe los esquemas de libre comercio capitalista. V.g. en adquisición de productos digitales: Wikipedia, enciclopedia en que la comunidad contribuye; Spotify y Youtube, que amenazaron la industria de la música y los derechos de autor.

Otro ejemplo que actualmente es novedoso se da con la adquisición de impresoras 3D individuales, ya que bajo la ideología del Código abierto, al comprar la impresora 3D libre y adquirir un diseño de tenis de código abierto, el usuario no tendría más la necesidad de comprar tenis de marca, porque podría imprimir esos tenis e incluso cambiarle el color. La práctica anterior rompería el sistema económico de consumo en bienes tangibles como lo conocemos.

Ahora bien, anteponer el trabajo o bien común por encima del capital es el común denominador de la economía colaborativa y las sociedades cooperativas, aunque puede ser que la administración interna sea diferente. Pero se denota indiscutiblemente que la cooperación es "hija de la necesidad"28 tanto en el siglo XIX como en el XXI. Por lo que, ambas, el cooperativismo y las economías colaborativas deben concebirse como parte de la economía social. ${ }^{29}$

En sus inicios, la economía social se comprendía en dos formas distintas: la primera, consistía en la filantrópica basada en el tutelaje del patrón sobre sus trabajadores, y la segunda, estaba vinculada al mutualismo y el cooperativismo. ${ }^{30}$ En la actualidad, la segunda visión parece ser la adaptable a las economías colaborativas.

Por lo anterior, la base conceptual y regulatoria de las economías colaborativas debe establecerse a partir de la economía social y el marco de principios y valores que ya proporcionan las economías cooperativas, sostenidos en tres

27 Rifkin, Jeremy, The marginal cost society, United States, Palgrave Macmillan, 2014, p. 11.

28 "La expresión se ha atribuido a H. Desroche, Le projet coopératif, París, 1976". Gadea Enrique, Cooperativismo y globalización, Universidad de Deusto, Asociación Internacional del Derecho Cooperativo, p. 61, disponible en: goo.gl/OjmPDR.

29 Cita a Castel, R., Les métamorphoses de la question sociale. Une chronique du salariatm, París, Fayard, 1995, en Forni Floreal, Freytes Ada, Quaranta Germán, "Frédéric Le Play: un precursor de la Economía Social" Instituto de Investigación en Ciencias Sociales, Facultad de Ciencias Sociales, Universidad del Salvador, abril de 200, pp. 6 y 14, disponible en: goo.gl/wOiAsF.

30 Spicker, Paul et al., "Pobreza: un glosario internacional", p. 109, disponible en: goo.gl/ hATVDb. 
grandes rubros: "trabajo antes que capital, incentivar al bien común y administración justa". ${ }^{31}$

Ahondando en ello, de acuerdo con las conclusiones del Congreso del Centenario de la ACI de Manchester de 1995, se distinguieron tres valores esenciales: "igualdad y equidad, autoayuda voluntaria y mutua, progreso económico y social". ${ }^{32}$ Mientras que las normas morales básicas que forman parte de la cultura cooperativa y espíritu colaborativo son: "la honradez, la preocupación, el pluralismo (enfoque democrático) y la capacidad de construir". ${ }^{33}$

De forma tal, que estos valores y normas morales han visto su reflejo en los Principios de las cooperativas, mismas que se pueden adaptar a las economías colaborativas, es decir: 1) asociación voluntaria y abierta, 2) administración democrática por los asociados, 3) justa distribución económica de los socios, 4) autonomía e independencia, 5) educación, formación e información, 6) cooperación entre cooperativas, 7 ) interés por la comunidad. ${ }^{34}$

por otro lado, la definición de economía social del CESE ayuda a identificar las dos vertientes en que se divide:

- La que navega en el subsector de mercado, obteniendo ganancias y realizando un reparto justo y equitativo de las mismas, ${ }^{35} \mathrm{y}$

- La del subsector del no mercado, que si bien comercializan lo hacen bajo precios simbólicos con el objetivo de ayudar a un bien social, o bien incluso aquellos sin lucro. ${ }^{36}$

La subdivisión anterior sirve de base para la regulación de las economías colaborativas orientadas al bien común, ya que coinciden en aquella clasificación de mercado y de no mercado. Es decir, primero las que buscan la generación de un lucro para la plataforma a través del cobro equitativo de gestión, y además promueven la colaboración entre los usuarios de la plataforma; v.g., Blablacar, Myt-

31 Monzón, Luis y Chaves, Rafael, "Es la economía social en la unión europea”, Informe elaborado para el Comité Económico y Social Europeo por el Centro Internacional de Investigación e Información sobre la Economia Pública, Social y Cooperativa (CIRIEC), 2006, p. 10, disponible en: goo.gl/ Y ₹94n4.

32 Charterina Martínez, Alejandro, "Los valores y principios cooperativos", op. cit., p. 7.

33 Idem.

34 De los cuales Paul Lambert expone una ampliamente en su obra La doctrina cooperativa, 1970, p. 272.

35 Pérez de Mendiguren et al., "¿De qué hablamos cuando hablamos de economía social y solidaria? Concepto y nociones afines”, XI Jornadas de Economía Crítica, Bilbao, 27-29 de marzo de 2008, p. 6, disponible en: goo.gl/ zYjGVh.

36 Idem. 
winpace, tutanda. Y segundo, con la finalidad de no pretender lucro y sólo servir de intermediario como el software libre y el código abierto, v.g. Wikipedia.

Por otro lado, se puede apreciar el éxito de sociedades cooperativas en el mundo gracias a las acciones privadas, de política pública y regulaciones, dado que algunos países aportan datos estadísticos del impacto en sus economías dejando ver que ayudan a la generación de empleos. ${ }^{37}$ De esta manera, las economías colaborativas pueden ir en la misma dirección.

Las experiencias internacionales positivas y la existencia de una política pública de economía social son el caldo de cultivo perfecto para lo que Scholz y otros autores postulan como la evolución de las cooperativas tradicionales en "plataformas cooperativas" o cooperativas 2.0, ${ }^{38}$ las cuales representan una oportunidad de desarrollo para la economía y beneficio social directo.

La política pública que impulsó el esquema cooperativo en México a pesar de haber tenido auge en el S.XIX y contar con resultados exitosos ${ }^{39}$ se desdibujó con el paso del tiempo. Es hasta el incipiente Programa de Fomento a la Economía Social 2015-201840 y la Ley de Economía Social que el tema figura de nuevo en la agenda pública; para este estudio toma relevancia puesto sirve de marco público para insertar una regulación dirigida a las economías colaborativas.

El retraso del tema de la economía social en la agenda pública del país ha implicado que a la fecha no se cuenten con datos estadísticos sólidos del impacto de las cooperativas en la economía mexicana. Apenas el INEGI ha iniciado un programa que ayude a conocer datos precisos de su impacto. ${ }^{41}$

37 Roelants, Hyungsik, Terrasi, Cooperativas y empleo: un informe mundial, Organización internacional de cooperativas en la industria y los servicios, CICOPA\&Grupo Desjardins, 2014, pp. 120-124, disponible en: goo.gl/8vrC8B.

38 Albert Cañigueral, Vivir mejor con menos, Conecta, 2014. En entrevista realizada por L'Apòstrof, publicada por Nexe, quaderns d' autogestió d' economia cooperativa, disponible en: goo. gl/Y8Wh6a.

39 Por ejemplo, en España Grupo Mondraggon, en México cooperativas como: Coop. Cruz Azul, Coop. Pascual, Coop. Trabajadores de Occidente, TOSEPAL. Ejemplos de cooperativas sociales de los estados y grupos indígenas se pueden encontrar en el "Diagnóstico del programa de fomento a la economía social” del Instituto Nacional de Economía Social, Universidad Iberoamericana de Puebla, disponible en: goo.gl/tcymXp.

40 Acuerdo por el que se aprueba el Programa de Fomento a la Economía Social 20152018, Diario Oficial de la Federación, 18 de junio de 2015, disponible en: goo.gl/9eSjfle.

41 Soto Tellez, Felipe, "Economía social y solidaria: reflexiones de una Política Pública", Alianza Cooperativista Nacional, 1o. de octubre de 2012, disponible en: goo.gl/ SOwH8B. 
Sin embargo, a consideración del autor, el gobierno mexicano está carente de una política pública de economía social a largo plazo, que prevea las economías colaborativas como parte de ese impulso para el desarrollo; fomentando mayor financiamiento y régimen legal especial, además de un claro método de identificación.

En suma, los conceptos que provee la economía social sirven como punto de partida para regular las economías colaborativas en México. Y de esa forma evaluar su actividad e impacto en la sociedad, ello para determinar si son en realidad economias colaborativas orientadas al bien común, o si pertenecen a las economías corporativas online P2P.

Consecuentemente, si se determina congruente la operación de una economía colaborativa orientada al bien común con los principios y valores de la economía social, dicha empresa debe ser acreedora a incentivos fiscales para desempeñar su actividad, ya que ayudan a resolver problemas sociales.

Por otro lado, si se determina que por su actividad la empresa en realidad obedece a un esquema corporativo online P2P del capitalismo salvaje, su trato debe orientarse al de una empresa tradicional que responda socialmente a su enriquecimiento desmedido a partir de los riesgos financieros, materiales y humanos de otros.

\section{MÉTODO PARA IDENTIFICAR UNA ECONOMÍA COLABORATIVA}

\section{Método de análisis para identificar la orientación de Plataforma P2P}

Para establecer un marco regulatorio que contenga los conceptos anteriores, se propone en los siguientes párrafos una metodología de análisis en torno a la naturaleza de las actividades de las plataformas como intermediarios.

El método de clasificación se ha diseñado con variables que ayuden a identificar la conducta de la empresa de acuerdo con sus valores, principios y desarrollo de actividades con los colaboradores, así como la relación que mantiene con la sociedad.

De esa forma, se logrará dilucidar qué tipo de empresa es, en el marco de la "economía colaborativa" en un amplio sentido, y por tanto, determinar a qué regulación debe ser sujeta. ${ }^{42}$ Ya que si es una empresa que se conduce con responsabilidad social se puede incentivar fiscalmente, a diferencia de una

\footnotetext{
42 Charterina Martinez, Alejandro, "Los valores y principios cooperativos", op. cit., p. 4.
} 
empresa que se enriquece a costa de la sociedad, entonces debe responsabilizarse con ella y fiscalizarse.

Para concluir un entendimiento razonado, lúcido y veraz sobre la naturaleza de una economía colaborativa de acuerdo con las contradicciones de la finalidad que persiguen y sus actividades; he diseñado cuestionamientos (véase cuadro 2) de acuerdo con los indicadores (véase cuadro 1) que permitirán arrogar una clasificación.

\begin{tabular}{|lll|}
\hline \multicolumn{4}{|c|}{ Cuadro 1. Indicadores para la clasificación de las economias colaborativas } \\
\hline 1) Principios y valores & 5) Prácticas Monopólicas $y$ & 8) Responsabilidad social \\
2) Actividades informáticas & Competencia desleal & 9) Protección de datos personales y \\
3) Desarrollo de mercado & 6) Sociedad y Cooperativas & 10) Cumplimiento fiscal. \\
4) Relación con los particulares & 7) Resolución de conflictos y & \\
& Protección al consumidor & \\
\hline
\end{tabular}

Así, las respuestas a las preguntas descritas en el cuadro 2 dotarán de información suficiente para clasificar la actividad de la plataforma. En este sentido, se encontrará, por ejemplo, que:

- Si la empresa se guía bajo principios de distribución inequitativa, un lucro excesivo y desproporcionado en términos porcentuales respecto del desgaste de los activos fijos de los particulares, creando un nicho de mercado y manteniendo el control sobre él, so pretexto de la intermediación tecnológica; además establece exclusividad de los socios inscritos, mantienen control de los pagos, realiza prácticas monopólicas o competencia desleal, niega la solución de conflictos, niega la protección al consumidor, no es responsable socialmente, no tiene control de datos personales y/o no contribuye fiscalmente. Entonces se está en presencia de una economía colaborativa corporativa y es en realidad una economía corporativa online P2P que requiere ajustarse a la regulación de una empresa tradicional, o

- Si se detecta que la empresa se guía bajo principios y valores equitativos de riqueza, no tiene fines de lucro o persigue lucro equitativo de acuerdo con el riesgo entre los particulares y la empresa; además utiliza la plataforma tecnológica sólo como intermediario, pudiendo crear o no crear un nicho de mercado, pero no ejerce control sobre el mismo; es decir, no tiene control de pagos, ni realiza prácticas monopólicas y además no niega la protección al consumidor (si es que la hay) y provee de solución de conflictos o no establece su negativa, protege datos perso- 
nales y contribuye o no fiscalmente de acuerdo con la actividad; entonces se está en presencia de una economía colaborativa orientada al bien común.

Así pues, el resultado del diagnóstico permitirá un nivel de clasificación que arroje la regulación legal apropiada y justa para la actividad de la plataforma.

Aunado al método anterior, la matriz de la economía del bien común, de Felber, ofrece una opción matemática para identificar la justicia social y la distribución de los beneficios en la empresa dentro de modelos de negocio del capitalismo salvaje. ${ }^{43}$ Mediante ésta se evalúa a las empresas de acuerdo con su forma de gestión en seis categorías: a) proveedores, b) financiadores, c) empleados, d) clientes, e) ámbito social, y f) negativos.

Cada uno de estos seis elementos se califica con un máximo de puntaje determinando por la matriz. De esa manera, el puntaje total arrogará un número final que se medirá dentro de una categorización de nivel.

Por razones de extensión del presente artículo resulta imposible explicar más. Empero, la relación de ambos métodos contribuyen a la rutas de algoritmos mediante los cuales se puede elaborar una plataforma automatizada que por producto final arroje una certificación o sello de confianza para las plataformas de economías colaborativas orientadas al bien común, de forma tal que operen con el visto bueno del Estado, generando confianza en los usuarios.

\section{Ejemplos casuísticos}

A continuación se ejemplificarán algunos casos bajo el análisis del método expuesto (véase cuadro 2), se aclara que un examen exhaustivo y detallado requería mayor extensión. Por lo cual se exponen las generalidades principales.

\section{A. Caso Uber}

Los valores y principios de Uber responden a una empresa corporativa online $P 2 P$, ya que antepone la generación de capital a la generación de trabajo, niega en sus contratos de Adhesión ${ }^{44}$ la existencia de la figura jurídica del empleo,

43 Felber, Christian, La economía del bien común, "la matriz de bien común 4.0", Deusto, 2012, pp. 58 y 59.

44 1) Contrato de modelo de porcentaje para tu chofer, 2) Contrato de modelo de renta de tu vehículo para el chofer, 3) Carta de comodato por el teléfono celular, 4) Contrato de confidencialidad, 5) Acuerdo de liberación laboral del chofer al socio, 6) Carta de Responsiva 
misma que cambia por "chofer"; ejerce un control total sobre precios y crea un nicho de mercado de transporte privado, así mismo niega caer en el nicho del servicio de taxis.

La recepción en México ha sido acogida por la necesidad de un mejor servicio de taxis; sin embargo, recibió presión social del gremio taxista bajo el argumento de la competencia desleal y la regulación excesiva del servicio de taxis.

Uber es una sociedad de responsabilidad limitada cuya fuente de riqueza se obtiene de la intermediación tecnológica que ofrece en territorio nacional, es decir, por el 25\% que cobra a sus contratistas. Y es la actividad por la que debería fiscalizarse. Uber está dado de alta en Hacienda y paga un impuesto gubernamental de 1.5\% aprobado en 2016 por la Ciudad de México. Pero esto no resuelve otros problemas: el del consumidor final y la resolución de conflictos, la protección de datos, la falta de responsabilidad social con los contratistas y choferes, ni que la empresa obtiene fuente de riqueza en territorio nacional.

\section{B. Airbnb}

Airbnb, es una sociedad constituida en Estados Unidos, India y China, "ofrece una plataforma por Internet que conecta a anfitriones que tienen alojamientos disponibles para anunciar y reservar con huéspedes que buscan reservar dichos alojamientos", "45 no así el servicio de alojamiento. Sin embargo, sí crea un nicho de mercado de rentas y alquileres de habitaciones, casas y departamentos por métodos de pago electrónicos.

No ejerce un control total del precio, empero al tenor de la dinámica de la oferta y la demanda, los costos actuales (2016-2017) de un hospedaje Airbnb son similares a la de hospedajes clasificados con estrellas, y estos alojamientos realizados por anfitriones corren el peligro de no contar con los mínimos de limpieza y seguridad de un hotel, aunque la elección de dicha opción tiene que ver más con la prioridad subjetiva de aquel que requiere el servicio.

"El gremio hotelero en México se considera afectado por este tipo de concentración monopólica de la oferta del servicio según lo comenta en entre-

del vehículo, 7) Carta de comodato del vehículo, Se especifican cantidades y costos de los siete tipos de contratos que van desde los doscientos pesos hasta cuatro cientos pesos. Se puede consultar Uber, Contratos para socios, op. cit.

45 Airbnb, Términos y condiciones legales, sitio web, disponible en goo.gl/ZAi9rg. 
vista el Director del Registro Nacional de Turismo". ${ }^{46}$ Es decir, no afecta que una persona decida rentar una habitación de forma aislada, pero la existencia de una plataforma que concentre esas y otras ofertas, representa un tipo de competencia.

El modelo de software de Airbnb si bien se dedica a interconectar personas, lo cierto es que la actividad secundaria de las grandes ganancias obtenidas es por los contratos de hospedaje mercantil que establece de facto y que sin su existencia no serían posibles, por lo cual, debería responder social y económicamente a los que ponen sus recursos materiales, financieros y humanos en riesgo.

Si bien, aquel que renta habitación, casa o departamento (anfitrión), no puede encuadrarse para el cumplimiento normas oficiales mexicanas que regulan la hotelería, sí es verdad que aquél que se dedica a la actividad frecuente de la renta o alquiler, debe pagar una contribución al Estado, lo cual no hacen los anfitriones. Éstos actúan bajo la figura jurídica del contrato de hospedaje en materia mercantil y ya que el contrato reúne todos los requisitos de validez y forma, por ello debería expedir factura, pero se acogen del modelo de negocio para no hacerlo.

Mientras que la fuente de riqueza de Airbnb, empresa extranjera, proviene de la intermediación tecnológica obtenida en territorio nacional de la cuota del anfitrión; y por tanto, la empresa debería pagar un impuesto sobre esa ganancia adquirida en el país. Al no estar regulada esa actividad en la Ley del Impuesto Sobre la Renta (ISR), Airbnb no encuadra en el tipo del artículo 158 y 159 que grava los ingresos obtenidos de residentes extranjeros por la actividad de hospedaje.

Entonces, aquél que hospeda obtiene riqueza por hospedaje y la plataforma la genera por la intermediación tecnológica; pero el que adquiere el servicio carece de protección de consumo, solución de conflictos y devolución efectiva.

El contrato de Airbnb excluye la cláusula de solución de conflictos y obliga a renunciar a la class action o demanda colectiva. Por lo cual, al consumidor final, es decir, el huésped, se le niega su derecho de protección al consumo, que en realidad es irrenunciable.

La problemática radica en que si un huésped tuviera algún problema con el anfitrión y acudiera a la Procuraduría Federal de Protección al Consumidor,

46 Entrevista a Rodrigo Artuto Brenan Illán, Director del Registro Nacional de Turismo de la Secretaría Nacional de Turismo, realizada por el autor, 19 de enero de 2017. 
ésta se declararía incompetente para actuar, ya que el contrato de Airbnb dicta que su servicio es de software. Entonces el huésped o consumidor tendría que llamar a la persona que le proveyó el servicio, pero si reside en el extranjero, el problema es de competencia y jurisdicción sobre la celebración del contrato.

Por otro lado, el anfitrión también está desprotegido contra abusos de usuarios; v.g., en caso de que el huésped no quiera abandonar el lugar, cause desperfectos, delitos, etc.; siendo así, la plataforma no se hace responsable. En suma, Airbnb, no es una economía colaborativa orientada al bien común.

\section{Blablacar}

Blablacar es una sociedad anónima constituida en Francia denominada COMUTO S.A., la cual facilita a través de medios tecnológicos la oferta de asientos desocupados para viajes largos o cortos en el auto de los usuarios que se inscriben y publican sus viajes.

El contrato de su uso estipula que la plataforma no se encarga de los pagos y que el precio del transporte no debe reflejar lucro alguno, el precio debe ser estrictamente sólo para la división de los gastos del viaje, como la gasolina y el desgaste del auto.

Si bien, se desarrolló un mercado no generador de monopolio, ni persigue fines de lucro desmedido, en su contrato cobra gastos de gestión, los cuales le permiten generar riqueza a COMUTO, por lo cual debería declarar sus ingresos y pagar ISR. Además, el contrato no asegura la protección de datos personales, ni ofrece sistema de solución de conflictos o protección al consumo.

Si bien, la plataforma tiene por objeto ayudar a solucionar conflictos de movilidad a precio justo y equitativo entre los partícipes, debería también elevar el nivel de seguridad de datos y ofrecer un sistema de solución de conflictos eficaz, además de declarar los ingresos generados en territorio nacional y pagar ISR. Y dado que su actividad es social podría incentivarse fiscalmente.

\section{PRopuesta de REgUlación DE LA ECONOMíA COLABORATIVA EN MÉXICO}

\section{Marco regulatorio para las economías colaborativas}

Los conceptos que provee la economía social sirven de punto de partida para regular las economías colaborativas en México. Y dado que un marco 
regulatorio responde a la visión de un Estado nación, el Estado mexicano se encuentra en la diatriba de: incentivar el capitalismo salvaje o decantarse por ser promotor de la economía social en este tema.

La base de la elección deberá responder a la sustancia de la norma constitucional desde un punto de vista de la ciencia jurídica. Aunque desde el punto de vista de la ciencia política será el resultado de las pugnas internas de los intereses de los actores económicos y políticos de un Estado Nación.

Una regulación bajo la visión del Estado de derecho social que sirva de contrapeso al modelo económico neoliberal debe partir de un concepto de "economía colaborativa" en sentido amplio, estableciendo los principios y valores que ofrece la economía social y la estructura de las cooperativas.

De forma tal que se permita identificar una economía colaborativa orientada al bien común y promover el desarrollo de plataformas cooperativas 2.0, distinguiéndolas de las aquí denominadas economías corporativas online P2P.

El objetivo principal para regular las economías colaborativas debe ser:

- Incentivar el desarrollo del trabajo antes que capital. Esto es, motivar los modelos de negocios que generen trabajos flexibles con condiciones justas bajo los estándares de la OIT, mismas que pueden gratificarse fiscalmente.

- Incentivar las actividades al bien común. Es decir, los modelos de negocios pueden desarrollar actividades que independientemente de su objetivo de lucro, ayuden a solucionar necesidades sociales, tal como movilidad urbana, turismo, medio ambiente, etcétera.

- Incentivar una administración justa y equitativa entre la empresa y los colaboradores del modelo de negocio, es decir, aquellos agentes que ponen en riesgo sus recursos humanos, materiales y financieros. Lo cual se puede lograr a través de beneficios fiscales.

Para realizar lo anterior, el Estado mexicano cuenta con fundamentos constitucionales y leyes federales que tienden a un Estado de derecho social e impulso de la economía social. Estos fueron manifiestos recientemente en la Ley de Economía Social y Solidaridad reglamentaria del artículo 25 párrafo octavo constitucional que establece los mecanismos para facilitar "la organización y la expansión de la actividad económica del sector social”. ${ }^{47}$

47 Constitución de los Estados Unidos Mexicanos, Diario Oficial de la Federación, 5 de febrero de 1917, última reforma publicada el 24 de febrero de 2017, p. 24, disponible en: goo.gl/ a8XOV9 (fecha de consulta: 1o. de mayo de 2017). 
El artículo 25 de la Constitución mexicana determina la rectoría del Estado en la economía, el párrafo primero textualmente señala lo siguiente:

\begin{abstract}
Corresponde al Estado la rectoría del desarrollo nacional para garantizar que éste sea integral y sustentable... que fortalezca la Soberanía de la Nación... mediante la competitividad, el fomento del crecimiento económico y el empleo y una más justa distribución del ingreso y la riqueza, permita el pleno ejercicio de la libertad y la dignidad de los individuos, grupos y clases sociales... ${ }^{48}$
\end{abstract}

Además el párrafo tercero del mismo artículo promueve la "responsabilidad social" de sector privado y público; el párrafo sexto establece que "impulsará a las empresas de los sectores social y privado de la economía, sujetándolos a las modalidades que dicte el interés público y al uso, en beneficio general, de los recursos productivos, cuidando su conservación y el medio ambiente". ${ }^{49}$

En esta tesitura, el Estado está obligado a regular las economías colaborativas como parte de la economía social, por tanto, puede adherir el multicitado concepto en el párrafo octavo constitucional, y crear un capítulo expreso dentro de la Ley de Economía Social y Solidaria antes referida. ${ }^{50}$

Así mismo, el Estado mexicano deberá fomentar un política pública de economía social a largo plazo, que fortalezca el Estado de derecho social y sirva de contrapeso al sistema económico capitalista imperante; de forma tal que motive a las organizaciones sociales a navegar en esquemas financieros y legales más amigables. Tal como se sustentará en los siguientes párrafos.

\title{
2. Política pública de economía social de largo plazo
}

El actual Programa de Fomento a la Economía Social 2015 -2018, si bien representa un avance loable, este autor considera que aún es un esfuerzo lacso y pobre por cuanto constituye una política pública de largo plazo que defina un Estado de derecho fortalecido en el tema. Además, el Estado está obligado

48 Ibidem, p. 25.

49 Idem.

50 Ley de la Economía Social y Solidaria, reglamentaria del párrafo octavo del artículo 25 de la Constitución Política de los Estados Unidos Mexicanos, en lo referente al sector social de la economía, Diario Oficial de la Federación, última reforma del 30 de diciembre de 2015, disponible en: goo.gl/UDhqyC. 
Esta revista forma parte del acervo de la Biblioteca Jurídica Virtual del Instituto de Investigaciones Jurídicas de la UNAM

a mostrar informes claros y públicos de los avances de dicho Programa, lo cual no hace.

Sin embargo, es de resaltar que dentro de las líneas estratégicas se encuentran trabajos a desarrollar sumamente interesantes que pueden fortalecerse a corto, mediano y largo plazo. En tal virtud, en este trabajo se proponen estrategias específicas (sin descalificar las ya planteadas en dicho programa), mismas que a continuación se describen.

\section{Propuestas legales específicas}

\section{A. Reforma a la Constitución}

Incluir el concepto "economías colaborativas" en el párrafo octavo del artículo 25 de la Constitución. El beneficio de estipular de forma concreta el concepto en el texto constitucional, no deja lugar a duda de la rectoría del Estado sobre el tema, aunque el mismo párrafo octavo cuando dice "cualquier forma de economía social" ${ }_{11}$ da cabida a la regulación reglamentaria.

\section{B. Reforma a la Ley de Economía Social y Solidaria}

Cuadro 3. Contenido del Capítulo

"Economías Colaborativas" en la Ley Reglamentaria

1) Establecerá los principios y valores que deben regirlas

2) Identificará las actividades informáticas de la P2P

3) Identificará el impacto y afectación en el mercado, así como las consecuencias de ello

4) Identificará la clasificación de la relación con los particulares y la generación de empleos

5) Establecerá consecuencias por Prácticas Monopólicas y Competencia desleal

6) Incentivará las Sociedades Colaborativas

7) Promoverá la Resolución de conflictos y Protección al consumidor

8) Incentivará la responsabilidad social

9) Dictará la protección de datos personales

10) Motivará gratificaciones fiscales

11) Incentivará contratos inteligentes (tecnología Blockchain) para dotar de seguridad al proconsumidor y consumidor

12) Incentivará devoluciones mediante tarjetas de crédito.

Incluir un capítulo general denominado "Economías colaborativas" en la Ley Reglamentaria, ello con la finalidad de dotar de los elementos que permi-

51 Constitución de los Estados Unidos Mexicanos, cit. 
tan identificar y regular las economías colaborativas orientadas al bien común de aquellas que no lo son, así como expresar los conceptos que ayudarán a determinar el proceder de esas empresas. El contenido de las directrices debe versar en los tópicos señalados en el método de este artículo (véase cuadro 3).

\section{Reforma a la Ley del Impuesto sobre la Renta (ISR)}

A partir de la conceptualización de la economía colaborativa debe realizarse una reforma a la Ley del Impuesto sobre la Renta que grave la riqueza obtenida por residentes extranjeros debido a las actividades de intermediación tecnológica con fines de lucro, el cual puede ser parte del Título V del Régimen de residentes extranjeros con ingresos provenientes de fuentes de riqueza ubicado en Territorio Nacional o elaborar un título especial de economías colaborativas.

A su vez, en el título VII de Incentivos fiscales, se debe motivar las actividades de las economías colaborativas sin fines de lucro como libres de gravamen, y establecer deducciones a las economías colaborativas con fines de lucro que promueven precios justos, colaboración y ayuden a resolver problemas sociales como el medio ambiente.

\section{Intervención del Estado y autorregulación}

Las propuestas legales se pueden abordar desde dos enfoques ideológicos. Primero desde la perspectiva de la intervención del Estado como una obligación del Estado de derecho social. Y segundo, desde la autorregulación.

Para tal efecto, se establecen dos propuestas respectivamente: a) la generación de un sello de confianza que cumpla con los requisitos de la economía social, ello a través de un ente público u organismo privado facultado por el Estado, y/o b) la resolución electrónica de disputas de forma privada, promoción y desarrollo de contratos inteligentes (blockchain) y un sistema de devolución efectiva (changer back).

a. Sello de confianza

La elaboración de un sello de confianza a través de un sistema automatizado con base en el método de análisis expuesto en el apartado anterior (véase cuadro 2), permitirá la implementación de una política pública y un marco 
regulatorio que dote de certeza jurídica e incentivos fiscales a las empresas que se constituyan de acuerdo a su actividad como economías colaborativas.

Este sello de confianza diseñado desde la visión de la economía social lograría certificar a las economías colaborativas y por exclusión no certificar a las empresas corporativas online P2P. La consecuencia es que los particulares que pretendan integrarse como socios a una plataforma podrán ver el nivel de puntaje de la empresa respecto de la economía social; lo cual tendrá una afectación directa sobre la reputación de la empresa.

Esto guiará al posible socio a evaluar el costo-beneficio real de formar parte de ese modelo de negocio, ya sea corporativo o colaborativo, basándose en la repartición de la riqueza a costa del riesgo de sus activos. La consecuencia será crear coacción social para las empresas corporativas, motivándolas a la responsabilidad social.

\section{b. Resolución electrónica de disputas}

Como formas de autorregulación se puede incentivar a las empresas a acudir o desarrollar sistemas de resolución de conflictos fiables y justos que actúen sin contravenir la protección al consumidor.

Además de promover el desarrollo de contratos inteligentes, en los que se establezcan variables que permitan a las partes obtener sus ganancias hasta que el pacto se haya cumplido de acuerdo con las variables determinadas en el contrato. De forma tal que, mientras no se cumplan las variables del contrato, las ganancias de ambos se conservarán retenidas. Aunque es justo mencionar que esta salida resulta un tanto utópica ya que infiere cambiar el sistema de compra-venta.

Por lo que promover un sistema de devolución efectivo es una salida pragmática viable en el sistema actual, en la cual se requiere la intervención bancaria y la regulación de estas acciones para garantizar la solución de conflictos.

\section{c. La interpretación de ley respecto de los contratos en México}

Las economías colaborativas establecen sus relaciones y actividades a través de contratos de adhesión, debido a que el acto jurídico se replicará miles o millones de veces, así el usuario no tiene margen de negociación. A partir de análisis de las cláusulas de los contratos en oposición a los hechos se puede desmitificar el contrato, ya que éste representa simplemente el dicho de la 
empresa. Y son los hechos los que dan el derecho aplicable y no el dicho, ya que un contrato no es ley.

En este sentido, aunque un contrato de intermediación insista en sus cláusulas en la inexistencia de relación laboral, mientras exista el elemento de subordinación y control de pago, cualquier juez mexicano dictará la existencia de la relación laboral. Por tanto, existe de forma directa e indirecta un lucro a partir del trabajo de otros, lo que supone la expresión pura del capitalismo.

La ley debe establecer el fundamento para diferenciar una economía colaborativa orientada al bien común (economía social) de una que no lo es, para dotar a los Tribunales Mercantiles y Laborales de un cuerpo legal que fortalezcan su interpretación en caso de un conflicto.

\section{d. Fortalecimiento del Observador (osse.org.mx)}

La línea estratégica del Programa que fomenta la Economía Social de la cual se desprende el Observador (osse.org.mx), puede llegar a constituirse en un instrumento de reputación y certificación para las economía sociales, incluyendo las economías colaborativas P2P orientadas al bien común.

e. Aportaciones desde la experiencia de derecho comparado en la Unión Europea

La propuesta de regulación de la Unión Europea respecto de economías colaborativas, provee de recursos a reflexionar para incorporarlas en una regulación en México.

Además, en un estudio, la Unión Europea reporta que la llamada economía colaborativa (no con la división que se presenta en este artículo) generó ingresos por 3,600 millones de euros en 2015 y que éstas se desarrollaron en cinco sectores clave, a saber: alojamiento (alquiler a corto plazo); transporte de personas; servicios para la vivienda; servicios profesionales y técnicos, y financiación colaborativa. ${ }^{52}$

El Comité Económico y Social Europeo (CESE) en su dictamen 2015, señala la complejidad del concepto economía colaborativa y la dicotomía con

52 "Todas las cifras se basan en estimaciones de PwC Consulting en el marco de un estudio contratado por la Comisión Europea", Comunicación de la comisión al parlamento europeo al consejo al comité económico y social europeo y al comité de las regiones, Una Agenda Europea para la economía colaborativa, op. cit. 
Esta revista forma parte del acervo de la Biblioteca Jurídica Virtual del Instituto de Investigaciones Jurídicas de la UNAM

la que han surgido entre las corrientes de pensamiento. ${ }^{53}$ Las contribuciones más importantes del CESE se reflejan en el numeral 19, al recomendar regular estos modelos de negocio ${ }^{54}$ sobre temáticas centrales que impactan el desarrollo de su actividad. Las cuales se presentan en forma de cuadro a continuación. ${ }^{55}$

Aunadas a estas recomendaciones, es necesario agregar una más: la resolución de conflictos. La ausencia de éste tópico se puede deber a la reciente Directiva de la UE sobre Resolución Electrónica de Conflictos.

\begin{tabular}{l} 
Cuadro 4. Aportaciones de la Unión Europea a la regulación de la \\
"Economías Colaborativas" \\
\hline 1) La legislación nacional y de la UE aplicable \\
2) Derechos de los trabajadores \\
3) Imposición fiscal adecuada \\
4) Derechos sociales \\
5) Protección de los datos y la privacidad de los participantes, \\
6) Competencia leal y la lucha contra los monopolios y las prácticas contrarias a la competencia; \\
7) Responsabilidad de las plataformas en que los negocios celebrados entre los socios y la legalidad \\
8) Protección de los derechos de consumo, incluyendo los socios que intervienen en la economía \\
colaborativa y también del prosumidor, mediante la adaptación a estas relaciones de todo el acervo \\
9) Disposiciones sobre las cláusulas abusivas, las prácticas comerciales desleales, salud, seguridad y \\
comercio electrónico; \\
10) Controlar el impacto ambiental de la economía colaborativa y prevenir sus repercusiones negativas \\
11) Derechos de autor y de propiedad intelectual.
\end{tabular}

Sin embargo, para México debe incluirse, ya que los contratos de algunas empresas corporativas P2P, como Airbnb, obligan a la renuncia del derecho de demandas colectivas, así como excluyen la cláusula de solución de conflictos; y dado que de acuerdo con su modelo de negocios no existe un consumidor, ya que es un modelo voluntario entre pares que deciden realizar un acuerdo; las agencias de protección al consumidor no tienen competencia para conocer de los asuntos.

53 Dictamen del Comité Económico y Social Europeo sobre el tema "La economía colaborativa y la autorregulación", Dictamen exploratorio, Ponente: Jorge Pegado Liz, 2016/C 303/05, disponible en: goo.gl/Fyle.94x.

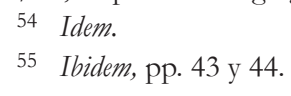




\section{DIMENSIÓN SOCIAL DE LA ECONOMÍA COLABORATIVA EN MÉXICO}

\section{México: caldo de cultivo para el capitalismo salvaje}

México vive el peor momento de crisis económica y política en su historia, ello aunado a la crisis medioambiental mundial que impacta de forma directa la economía en el alza de precios, la devaluación del peso, ${ }^{56} \mathrm{el}$ desempleo y la baja del poder adquisitivo, aunado al contexto internacional de las políticas del presidente estadounidense Donald Trump que impactan en pérdidas de empleos en el país; todo esto consecuentemente ha generado un clima de descontento social nacional.

Los datos del INEGI 2016 registran que la tasa de desocupación es de 3.5\%, lo que implica que 4 de cada 100 mexicanos no tienen una ocupación; además indican una tasa de subempleo de $7.1 \%$, lo que significa que 7 de cada 100 mexicanos tienen una subocupación. Estos datos sugieren que en México va muy bien el tema del empleo, pero esto no es así; lo que sucede es que las personas desempleadas obtienen refugio en la informalidad. Esto se confirma con la tasa de informalidad laboral de 52.4\%, lo cual indica que 6 de cada 10 empleos pertenecen a la economía informal. ${ }^{57}$

En este contexto, la no regulación de empresas corporativas P2P, el desinterés o la falta de comprensión del impacto social por parte del Estado en términos de los derechos sociales, aunado al clima político y la necesidad existente, insinúa que México es caldo de cultivo fértil para la explosión de empresas corporativas P2P.

Ante este posible, inminente y palpable escenario es urgente la necesidad un Estado de derecho social que motive economías colaborativas orientadas al fin común a través de beneficios fiscales. El gobierno mexicano está obligado a incentivar fiscalmente a pequeños y medianos productores nacionales, así como a empresas cooperativas nacionales que fortalezcan la economía y empleos tan necesarios, además de motivar el comercio transfronterizo a partir de los demás tratados de libre comercio firmados por el país.

56 El 5 de enero de 2015 el valor del peso frente al dólar era $\$ 14.9469$, el 5 de enero de 2016 el valor ascendió a \$17.3456 y el 5 de enero de 2017 el peso se devaluó a \$21.3799, alcanzando su máximo el 19 de enero de 2017 en $\$ 21.9094$, Información en el sitio web del Banco de México, disponible en: http:// wmw.banxico.org.mx/portal-mercado-cambiario/.

57 Ocupación y empleo, Instituto nacional de Estadística y Geografía, disponible en: goo.gl/ftf6Qc. 


\section{Economías colaborativas: opción pragmática a conflictos sociales}

El costo marginal cero y el código abierto son elementos contundentes en la práctica para las verdaderas economías colaborativas, puesto que los individuos colaboran para obtener beneficios. La gran contribución de estas formas económicas radica en que solucionen conflictos sociales y medioambientales, etcétera. Y en este sentido, el Estado debería motivarlas ya que por sí solo no puede manejar la diversidad de crisis sociales, económicas y sociales que confluyen.

Por ejemplo, pensemos en el alza de la gasolina en México. El 1o. de enero de 2017, los mexicanos despertaron con la noticia del alza de precio en la gasolina de casi $20 \%$ y el descontento social en las calles y en las redes sociales fue evidente. ${ }^{58}$ Ésta es una variable económica que afecta de manera directa los hogares de los mexicanos, puesto que sube el costo de transportación y los insumos comerciables que se transportan; entonces la familia debería reducir sus costos de transportación ¿Se puede esbozar una posible solución?

Pensemos en una plataforma de transporte colaborativa cuya única función sea ayudar a economizar el gasto en transportación, por ejemplo: en personas que van a los mismos lugares (trabajo/escuela) con horarios determinados y viven cercanamente. Si además, la plataforma y sus usuarios son gratificados fiscalmente se solucionaría un problema de economía familiar, movilidad y medio ambiente de la ciudad.

Por ejemplo, en un pequeño piloto en una escuela primaria, organizado con los padres de familia. La plataforma tendría que ayudar a automatizar la calendarización de los viajes, identificar a las personas, proteger los datos personales y garantizar la seguridad de la información. Los padres de familia deberían comprometerse a realizar los viajes alternadamente y a informar de la llegada segura de los menores, así como a tener seguro de sus unidades de transporte y responder en casos de emergencia.

El monto del precio del viaje debe ser acordado por los participantes, éstos podrían trabajar en grupos integrados fijos que acuerden que no habrá precio, porque habrá rotación circular de los padres, es decir, una vez cada uno, hasta completar el círculo.

$\mathrm{O}$ bien, pueden acordar un precio justo por viaje pensando en el desgaste del activo fijo y la gasolina. Así que el programa debería ayudar a calcular

58 Patiño Dainzú, Meana Sergio, "Precios de gasolinas subirán hasta 20\% a partir de enero", Economía, El Financiero, 27 de diciembre de 2016, disponible en: goo.gl/ kfq376. 
las variables en la relación del precio de la gasolina, distancia, tránsito y desgaste del auto. Este mismo caso se podría replicar a través de plataformas de comunicación sincrónica y asincrónica en lugares de trabajo, oficinas y universidades.

En suma, estaríamos en presencia de una verdadera economía social y solidaria que ayudaría a crear una conciencia de colaboración en los niños y adultos del futuro, quienes forjarán una sociedad de trabajo compartido y solidaridad más allá del sentido de propiedad. Lo cual ayudará a salir de un sistema salvaje de capitalismo que ha dañado a la sociedad y al medio ambiente provocando que vivamos los efectos del cambio climático y los destellos de la futura extinción de la humanidad.

\section{CONCLUSIÓN}

La Revolución industrial y el capitalismo salvaje imperante en la economía actual han provocado efectos negativos en la naturaleza y en la sociedad, v.g., carencia, desempleo y cambio climático. Hoy el Internet de las cosas en donde todo está conectado (casas, autos, electrodomésticos) es el futuro presente; es una nueva revolución tecnológica que de perpetuar el sistema económico tal como está será devastador para la humanidad y el mundo. Por lo que una justa medida de colaboración y riqueza es urgente sea impulsada por todos los actores, económicos, políticos y sociales.

Los beneficios de impulsar la economía colaborativa orientada al bien común apuntalan a transformar la convivencia de la sociedad, superando el concepto de propiedad privada por el de propiedad común, generando un impacto futuro en la solución de conflictos sociales, problemáticas ambientales y la vida humana en el planeta. Entonces, ¿qué México imaginamos en 50 años?

Un México en el que los negocios tecnológicos se desarrollen bajo medidas justas. Por ello, es urgente una política pública nacional a largo plazo que fomente la economía social e incluya las economías colaborativas para su identificación y motivación, inspirando un trato justo por cuanto a los porcentajes de riesgo, responsabilidad social y fiscalización. Además de un sello de confianza que las avale y califique mediante un sistema de puntaje y reputación. Lo anterior se logrará con las reformas propuestas, vertidas en resumen en el siguiente cuadro. 
Esta revista forma parte del acervo de la Biblioteca Jurídica Virtual del Instituto de Investigaciones Jurídicas de la UNAM

\section{Propuestas legales}

1. Política Pública Nacional de Economía Social a largo plazo (Reforma Constitucional)

2. Regulación Federal sobre Economías colaborativas (Reforma a la Ley de Economía Social)

- Facultar una Comisión u órgano privado o público certificador de sello de confianza, cuyo trabajo de forma simple se puede realizar mediante un sistema automatizado de análisis de identificación.

Trabajo en conjunto con el Instituto Nacional de la Economía Social de la Secretaria de Economía

Fortalecimiento del Observador(osse.org.mx)

Diseñar el sistema de clasificación de economías colaborativas con base en la metodología propuesta

Elaborar Manual de método de análisis de las plataformas

3. Reformas a la Ley de Protección al Consumidor para regular transacciones electrónicas en relación con las economías colaborativas corporativas.

4. Reformas a la Leyes Fiscales, incluir en ellas el concepto, economías colaborativas.

5. Reforma al Código Civil Federal y al Código Mercantil para la constitución de economías colaborativas.

\section{BIBLIOGRAFÍA}

\section{Libros y articulos}

Bonilla Egido, Antonio y Meler Playan, Javier, “Aplicaciones Distribuidas: P2P", Conceptes avançats de sistemes operatius, Departament d'Arquitectura de Computadors, 2016, disponible en: goo.gl/wvll0X.

Charterina MartíneZ, Alejandro, "Los valores y principios cooperativos", Dialnet, 1995, disponible en: goo.gl/TBW qeh.

Wosskow, Debbie, Unlocking the Sharing Economy, Reporte Independiente, Department for Business, Innovation \& Skills, Reino Unido, 2014, disponible en: goo.gl/yJmjDL.

FAirfield, Joshua A. T., "Smart Contracts, Bitcoin Bots, and Consumer Protection", Washington \& Lee University, Rev. Online, 2014, disponible en: goo.gl/ GfEpGY.

Farronato Chiara, Levin Jonathan, "The Rise Peer to Peer Bussines”, The Sharing Economies, Global Investor 2.15, noviembre de 2015.

FELBER, Christian, La economía del bien común, "la matriz de bien común 4.0", Deusto, 2012. 
FORNI, Floreal et al., Frédéric Le Play: un precursor de la economía social, Instituto de Investigación en Ciencias Sociales, Facultad de Ciencias Sociales, Universidad del Salvador, abril de 2000, disponible en: goo.gl/ $w$ OiAsF.

GADEA, Enrique, Cooperativismo y globalización, Universidad de Deusto, Asociación Internacional del Derecho Cooperativo, 2015, disponible en goo. gl/OjmPDR

Gromoslav Mladenatz, Historia de las doctrinas cooperativas, cooperativas, trad. de Carmelo Tumino, Buenos Aires, Intercoop, 1969.

Oram, Andy, Peer to Peer: Harnessing the Power of Disruptive Technologies, marzo de 2001, p. 8, disponible en: goo.gl/SwkGFl.

PÉrez de Mendiguren, Etxezarreta, Guridi, “¿De qué hablamos cuando hablamos de Economía Social y Solidaria? Concepto y nociones afines", XI Jornadas de Economía Crítica, Bilbao, 27-29 de marzo de 2008, disponible en: goo.gl/zYjGVh.

RIFKIN, Jeremy, The Marginal Cost Society: the Internet of Things, the Collaborative Commons, and the Eclipse of Capitalism, United States, Palgrave Macmillan, 2014.

ROELANTS et al., Cooperativas y empleo: un informe mundial, Organización internacional de cooperativas en la industria y los servicios, CICOPA\&Grupo Desjardins, 2014, disponible en: goo.gl/8vrC8B.

SAToshi, Nakamoto, Bitcoin: A Peer-to-Peer Electronic Cash System, 2014, disponible en: https:// bitcoin.org/bitcoin.pdf.

SCHOLZ, Trebor, Cooperativismo de plataforma. Desafiando la economía colaborativa corporativa, En Defensa del Software Libre, 2017, disponible en: https:// endefensadelsl.org.

SCHOLZ, Trebor, Cooperativismo de plataforma. Desafiando la economía colaborativa corporativa, Barcelona, Publicaciones Dimmons, Universitat Oberta de Catalunya, 2016, disponible en: goo.gl/TN8u1S.

Soto TelLeZ, Felipe, "Economía social y solidaria: reflexiones de una Política Pública", Alianza Cooperativista Nacional, 01, octubre de 2012, disponible en: goo.gl/SOwH8B.

SPICKER, Paul et al., Pobreza: un glosario internacional, Clacso, 2001, disponible en: goo.gl/ $h A 7 V D b$.

STALLman, Richard, "The GNU Operating System and the Free Software Movement", Open Sources: Voices from the Open Source Revolution, enero de 1999, disponible en: goo.gl/6uA2XG. 
Reportes y leyes

"Diagnóstico del programa de fomento a la economía social", Instituto Nacional de Economía Social, Universidad Iberoamericana de Puebla, disponible en: URL goo.gl/tcymXp.

Acuerdo por el que se aprueba el Programa de Fomento a la Economía Social 2015-2018, Diario Oficial de la Federación, 18/06/2015, disponible en: goo. gl/9eSjfk.

Comunicación de la comisión al parlamento europeo al consejo al comité económico y social europeo y al comité de las regiones, Una Agenda Europea para la economía colaborativa, Bruselas, 2 de junio de 2016, disponible en: goo.gl/CSF77r.

Constitución de los Estados Unidos Mexicanos, Constitución publicada en el Diario Oficial de la Federación el 5 de febrero de 1917, (texto vigente), última reforma publicada el 24 de febrero de 2017, disponible en: goo.gl/a8XOV9 (fecha de consulta: 1o. de mayo de 2017).

Dictamen del Comité Económico y Social Europeo sobre el tema «La economía colaborativa y la autorregulación, Dictamen exploratorio, Ponente: Jorge Pegado Liz, 2016/C 303/05, disponible en: goo.gl/Fyk.94x.

Ley de la economía social y solidaria, reglamentaria del párrafo octavo del artículo 25 de la constitución política de los estados unidos mexicanos, en lo referente al sector social de la economía, Diario Oficial de la Federación, última reforma del 30 de diciembre de 2015, disponible en: goo.gl/UDhquC.

Monzón, Luis y Chaves, Rafael, "Es la economía social en la unión europea", Informe elaborado para el Comité Económico y Social Europeo por el Centro Internacional de Investigación e Información sobre la Economía Pública, Social y Cooperativa (CIRIEC), 2006, disponible en: goo.gl/Y ₹94n4.

Peer To Peer Network, Gobierno de Hong Kong, Administración especial de la región, febrero de 2008, disponible en: goo.gl/7xZjTm.

Sitios web

Airbnb, Términos y condiciones legales, disponible en: goo.gl/ZAi9rg.

Banco de México, sitio web, disponible en: http:// wmm.banxico.org.mx/portal-mercado-cambiario/.

Blablacar, Términos y condiciones legales, sitio web, disponible en: goo.gl/ZO$k h b N$. 
Demanda colectiva Uber, sitio web, disponible en: http:/ / uberlawsuit.com/.

Diccionario de la Real Academia de la Lengua Española, disponible en: goo.gl/yWimis.

Diccionario de la Real Academia de la Lengua Española, disponible en: goo.gl/knBeNr.

Diccionario de la Real Academia de la Lengua Española, disponible en: versión electrónica en goo.gl/FNIWYK.

https:/ / freenetproject.org/.

Mytwinplace.com, Términos y condiciones legales, disponible en: goo.gl/KnFGxx.

Ocupación y empleo, Instituto nacional de Estadística y Geografía, disponible en: goo.gl/ftf6Qc.

Tutanda.com, Términos y condiciones legales, disponible en: goo.gl/FSLz9j.

Uber, Contratos para socios, disponible en: goo.gl/jHV $z^{5 Z}$.

Video en Entrevista, Jhon Duda, Plataform Cooperativism, The Internet, Ownership, democracy, the new schoool, November 13-14, disponible en: https:/ / vimeo. $\mathrm{com} / 149401422$.

\section{Hemerografia}

IsAAC, Mike y SINGER, Natasha, "California Says Uber Driver Is Employee, Not a Contractor”, New York Times, 17 de junio de 2015, disponible en: goo. $g l / 1 q 5 r J r$.

PatiÑo Dainzú, Meana Sergio, "Precios de gasolinas subirán hasta 20\% a partir de enero", Economía, El Financiero, 27 de diciembre de 2016, disponible en: goo.gl/ kfq376.

\section{Entrevistas}

Entrevista a Rodrigo Arturo Brenan Illán, Director del Registro Nacional de Turismo, realizada por el autor, 19 de enero de 2017.

Entrevista realizada por L'Apòstrof, publicada por Nexe, quaderns d' autogestió d' economia cooperativa, disponible en: goo.gl/Y8Wh6a. 